\title{
Del mercado único a la Unión Monetaria: ¿hacia una política económica común?*
}

\author{
Javier Bilbao \\ Profesor de la Universidad del País Vasco \\ Departamento de Economía aplicada
}

\begin{abstract}
Sumario: I. Introducción.-II. La arquitectura de gobernanza económica europea hasta la UEM.-III. La construcción de la UEM: opciones institucionales y de política económica. - IV. Los cambios en la gobernanza económica durante la gestión de la crisis. - V. Balance y conclusiones. VI. Bibliografía.
\end{abstract}

Resumen: La evolución de la gobernanza económica europea no ha sido lineal, sino que se han experimentado unos saltos cualitativos en la atribución de competencias para la intervención económica entre las instituciones comunitarias y las nacionales. Estos cambios de naturaleza estructural nos habrían acercado aparentemente a una política económica común a través de 3 etapas: Mercado común, Comunidad económica y Unión monetaria. Sin embargo, se ha llegado a un complejo y asimétrico sistema de interrelaciones que estaría reflejando que el denominador común del proyecto europeo ha sido la construcción plena del Mercado interior, para lo que se adoptaron todas las medidas consideradas necesarias, aunque supusieran compromisos adicionales en determinadas políticas económicas. Por eso puede afirmarse que se ha subordinado el proyecto de construcción europeo - y sobre todo su dimensión social - a las metas económicas compartidas.

Palabras clave: Gobernanza económica europea, Políticas comunes, Unión económica y monetaria, Economía política

Abstract: Rather than linear, the evolution of European economic governance has experienced some qualitative jumps in the conferring of competences for economic intervention between common and national institutions. These structural changes have ostensibly brought Member States closer to a common economic policy by means of 3 stages: Common Market, Economic Community and Monetary Union. However, a complex and asymmetric system of interrelationships has been developed revealing the common denominator of the European project to have been the complete construction of the internal market, and all measures considered necessary have been implemented to this end, although provoking additional commitments in different economic policies. Thus it can be said that the European construction project-particularly its social dimension-has been subordinated to some shared economic goals.

Keywords: European economic governance, common policies, Economic and Monetary Unión, political economy.

* Recibido el 20 de diciembre de 2013, aceptado el 7 de enero de 2014. 


\section{Introducción}

El proceso de definición de la arquitectura de gobernanza económica en la UE ha sido progresivo, aunque no lineal, y pueden identificarse algunos saltos cualitativos o discontinuidades en la atribución de competencias para la intervención económica entre las instituciones comunitarias y las de los Estados miembros. Estos cambios estructurales habían coincidido normalmente con etapas expansivas del ciclo económico (Consejo Económico y Social español, 2012, p. 18), hasta que la crisis —en la que aún estamossometió a la economía e instituciones europeas a tales desafíos que ha forzado la adopción de un alud de importantes decisiones con un carácter defensivo y a menudo improvisado, y con discutida eficacia o consistencia.

En todo caso, a medida que se ha avanzado en el proceso de integración, se ha ido perfilando una mayor interacción o integración política, aún con notables resistencias por parte de muchos de los estados, que se ha reflejado en la gobernanza económica. Es cierto que en esta evolución ha predominado el intergubernamentalismo y que, como señala Van Middelaar (2013), también han prevalecido tanto una visión funcionalista del proceso en los despachos de Bruselas como una permanente provisionalidad de las decisiones que nos ha llevado a un estado de transición permanente.

Pero, si tenemos que analizar la evolución de la arquitectura de gobernanza económica europea, es conveniente distinguir tres etapas fundamentales:

- Una primera etapa, que podemos identificar como la fase de construcción del Mercado común, y que iría desde el momento de la firma de los Tratados de París (1951) y Roma (1957) - que conforman los «Tratados Constitutivos» de las Comunidades Europeashasta la adopción del Informe Delors ${ }^{1}$ (1989).

- Una segunda etapa, coincidente con la fase de concepción y posterior puesta en marcha de la Unión económica y monetaria (UEM), que exacerbó la necesidad de coordinación, y, por eso, fueron surgiendo nuevos acuerdos y recursos de gobernanza económica europea a partir de 1990, entre los que destaca el Tratado de Maastricht de 1992.

- Una tercera etapa, forzada por la irrupción de la crisis a partir del segundo semestre de 2007, que ha condicionado absolutamente la gobernanza económica en la UE y ha conducido a la adopción de numerosas y relevantes decisiones -insisto en que no siempre consistentes - cuyas implicaciones han pasado desapercibidas para gran parte de la opinión pública.

${ }^{1}$ Libro Blanco Crecimiento, competitividad y empleo. Retos y pistas en el siglo XXI (Comisión de las Comunidades Europeas, 1993). 
En el resto del artículo trataré de sintetizar lo más relevante de estas etapas y de interpretar el significado de las mimas, desde la perspectiva de su contribución a la definición de una eventual política económica común, aportando una valoración personal y crítica de esta trayectoria en el apartado de conclusiones.

\section{La arquitectura de gobernanza económica hasta la UEM}

En esta primera etapa, se va consolidando en la UE una Política comercial común, a la vez que se avanzaba en el logro de un mercado común libre de obstáculos a la circulación de bienes y servicios. Es cierto, como señala Mariscal (2005), que ya el Tratado de la Comunidad Económica Europea (TCEE) de 1957 se refería al acercamiento progresivo y la coordinación de la política económica e incluso monetaria de cada uno de los Estados miembros (arts. 2, 3g, 105 y 145 del TCEE). Así mismo, dicho Tratado de Roma consideraba la política coyuntural de los Estados miembros como «una cuestión de interés común» (art. 103.1) e instaba la constitución de un Comité monetario de carácter consultivo (art. 105.2). Pero no es menos cierto que esas previsiones estaban orientadas exclusivamente a facilitar el equilibrio comercial (art. 104²), mediante el favorecimiento de la estabilidad cambiaria, en un proyecto que nacía con vocación de «mercado común».

Con ese objetivo de acercar y coordinar las políticas económicas de los Estados miembros, comienzan a constituirse, a partir de 1960, mecanismos de cooperación entre las administraciones nacionales - el Comité de políticas de coyuntura (1960), el Comité de política presupuestaria (1961), el Comité de políticas de medio plazo ${ }^{3}$ (1964) o el Comité de Gobernadores de Bancos centrales (1965) - que se saldarán con un grave fracaso en los intentos de coordinación, por los diferentes planteamientos de política económica de cada Estado miembro y por la falta de incentivos visibles para llegar a acuerdos (Ghymers, 2001).

Por el contrario, destacan, en este periodo, los acuerdos alcanzados para favorecer la estabilidad cambiaria (importante tras la paulatina adopción de los tipos de cambio flexibles en el sistema monetario internacional a princi-

2 «Cada estado miembro actúa la política económica necesaria para garantizar el equilibrio de su balanza de pagos global ya mantener la confianza en su propia moneda, teniendo cuidado de asegurar un alto nivel de ocupación y la estabilidad del nivel de precios» (Traducción del autor).

${ }^{3}$ Los 3 primeros comités mencionados fueron fusionados, en 1974, en el Comité de política económica por decisión del Consejo. 
pios de los años 70), mediante la coordinación de las políticas monetarias. En primer lugar, se creó la «Serpiente Monetaria Europea» (1972), que dará paso - en 1979 - al Sistema Monetario Europeo (SME). El SME era formalmente una serie de acuerdos bilaterales entre los bancos centrales orientados a limitar las oscilaciones máximas de cualquiera de las monedas de los Estados miembros; pero implicaba la necesidad de debatir colegiadamente sobre aquellas políticas nacionales que provocaban tensiones en los tipos de cambio e incorporaba un factor disciplinante (o una restricción) para las autoridades de cada Estado.

El SME, que implicó la creación del ecu como cesta de monedas comunitarias y un mecanismo de cambio e intervención - el Mecanismo de Tipos de Cambios (MTC) - , se fue perfeccionando técnicamente, pero también sufrió las presiones del mercado de divisas en 1992 y 1993, obligando a varios realineamientos de monedas (o a la salida temporal de varias de ellas, como la libra y la lira) y a ampliar las bandas de fluctuación (que pasaron del 2,25\% para las monedas más estables - o del $6 \%$ para lira, libra, peseta y escudo - a bandas del $15 \%$ ).

También en este período surgen las únicas políticas sectoriales comunitarizadas:

- En 1962 surge la Política agrícola común (PAC), orientada entonces a asegurar el autoabastecimiento de los ciudadanos comunitarios a precios asequibles, y a mejorar las condiciones de vida de las zonas rurales. Para ello se debía garantizar que el conjunto de países de la UE dispusiera de un sector primario viable mediante la mejora de la productividad, favoreciendo la reestructuración de las producciones. Las organizaciones comunes de mercados (OCM) y los precios de garantía o retirada, juntos con los fondos FEOGA, serán los pilares de la PAC, que absorbió en esta etapa más de la mitad de los recursos del Presupuesto europeo.

- La política pesquera común (PPC), cuyos orígenes datan de octubre de 1970, se basa también en las OCM. Los objetivos de la PPC son: la explotación sostenible de los recursos marinos, el aseguramiento de una renta para los pescadores, y el suministro regular de los consumidores y de la industria de transformación a unos precios razonables.

Para el resto de sectores económico estará vigente la Política industrial europea (PIC), con sus dos grandes metas: por un lado, la profundización del mercado interior, y, por otro, el logro de altos niveles de competencia intracomunitaria, mediante la fiscalización tanto de las políticas industriales nacionales (persiguiendo las ayudas estatales) como de los acuerdos colusorios entre las empresas. La PIC dará un salto en 1984 con la aprobación del Primer Programa-Marco de I+D comunitario, que da comienzo a la Política 
de innovación comunitaria, que tendrá una creciente participación en el Presupuesto de la UE.

Aprovechando la bonanza económica de la segunda mitad de los 80 , y el ambiente optimista por las recientes ampliaciones de las Comunidades europeas, se aborda el primer cambio relevante de la legislación primaria comunitaria: El Acta Única Europea, firmada en Luxemburgo y La Haya el 17 y el 28 de febrero de 1986, respectivamente, y que entró en vigor el 1 de julio de 1987, pretendió superar el objetivo de Mercado Común (Common market) para alcanzar el objetivo de Mercado Interior (Single market), que implicaría un espacio sin fronteras interiores, en el que la libre circulación de mercancías, personas, servicios y capitales estaría garantizada a partir de 1993. También se ampliaron las competencias comunitarias, admitiendo además que el Consejo pudiese tomar decisiones por mayoría cualificada, frente a la unanimidad que regía hasta entonces. Se incluía, además, el uso por primera vez del concepto de cohesión económica y social y la necesidad de disponer de fondos estructurales suficientes para su desarrollo (Consejo Económico y Social español, 2012, p. 16)

Con este nuevo impulso, en 1987 surge también un procedimiento de vigilancia multilateral de las políticas económicas, y lo que fue inicialmente un proyecto de «mercado común» comenzaba a adquirir perfiles de una «comunidad o unión económica», dando paso a una nueva etapa.

\section{La construcción de la UEM: opciones institucionales y de política económica}

En junio de 1988 el Consejo Europeo confirmó el objetivo de lograr progresivamente una UEM, y orientado a tal objetivo se constituyó un Comité - presidido por el entonces presidente de la Comisión Europea, Jacques Delors - para que estudiase y propusiera fases concretas para avanzar hacia dicha Unión. El fruto de la labor del Comité, el Informe Delors, aprobado en la Cumbre de Madrid de 1989, proponía un enfoque «gradualista» en virtud del cual la UEM se alcanzaría en tres fases. Aplicando estas recomendaciones, el Consejo Europeo de junio de 1989 acuerda el establecimiento de la UEM como un proceso de carácter progresivo que debía nacer en 1990, y en el que se distinguen tres fases ${ }^{4}$ :

\footnotetext{
${ }^{4}$ Para la realización de las fases segunda y tercera, era preciso revisar el Tratado de Roma, con objeto de establecer la infraestructura institucional necesaria. Con este fin, se convocó una Conferencia Intergubernamental sobre la UEM, que tuvo lugar en 1991, paralelamente a la Conferencia Intergubernamental sobre la Unión política. Las negociaciones concluyeron con la adopción, en diciembre de 1991, del Tratado de la Unión Europea, que fue
} 


\section{Primera fase (1990-1993)}

En esta fase se suprimen las limitaciones al movimiento de capitales entre los Estados miembros y se refuerza la cooperación entre los bancos centrales de los países, estableciéndose la libre utilización del ecu. El Tratado de Maastricht prevé ya la supresión de las monedas nacionales por la moneda común y también fijaba una serie de condiciones económicas de convergencia nominal, denominadas criterios de convergencia, relativas a la estabilidad de los precios, al déficit público, a la deuda pública, a los tipos de cambio y a los tipos de interés a largo plazo, criterios que eran de obligado cumplimiento para los países que quisieran integrarse en la UEM. Es el primer precedente exigente de vigilancia multilateral de las políticas económicas de los Estados miembros.

\section{Segunda fase (1994-1998)}

En este intervalo se crea el Instituto Monetario Europeo (IME), fundado el 1 de enero de 1994, como predecesor del Banco Central Europeo (BCE) y cuyas dos funciones principales eran: por un lado, fortalecer la cooperación entre los bancos centrales y la coordinación de las políticas monetarias; y, por otro, realizar los trabajos preparatorios necesarios para la constitución del Sistema Europeo de Bancos Centrales (SEBC), para la ejecución de la política monetaria única y para la creación de una moneda única en la tercera fase.

A propuesta del IME, en diciembre de 1995, el Consejo Europeo acordó que la unidad monetaria europea que habría de introducirse al inicio de la tercera fase se denominaría «euro», y confirmó que dicha fase comenzaría el 1 de enero de 1999. En diciembre de 1996, el IME presentó las series de los diseños de los billetes que entrarían en circulación en 2002. Además, el IME elevó al Consejo Europeo un informe que sentaba las bases para la creación del nuevo mecanismo de cambio (MTC II) - regulando las relaciones monetarias y cambiarias entre los países de la zona del euro y los demás países de la UE - que se adoptó formalmente en junio de 1997.

Cumpliendo las previsiones establecidas en el Tratado de Maastricht, el Consejo Europeo adoptó, en junio de 1997, el Pacto de Estabilidad y Crecimiento (PEC) cuya finalidad sería garantizar la disciplina presupuestaria en

firmado en Maastricht el 7 de febrero de 1992. No obstante, debido a los retrasos ocurridos en el proceso de ratificación, no entró en vigor hasta el 1 de noviembre de 1993. El nuevo Tratado modificó el Tratado constitutivo de la Comunidad Económica Europea, que pasó a denominarse Tratado constitutivo de la Comunidad Europea, e incorporó, entre otros, el Protocolo sobre los Estatutos del Sistema Europeo de Bancos Centrales y del Banco Central Europeo y el Protocolo sobre los Estatutos del Instituto Monetario Europeo. 
relación con la UEM. Al mismo tiempo, se irá haciendo efectiva la prohibición de los bancos centrales de financiar a las Administraciones públicas a través de las leyes nacionales que consagran su autonomía funcional (desarrollando las previsiones del art. 104.1 del Tratado de Maastricht).

En mayo de 1998 se adoptaron varias medidas importantes:

- El 2 de mayo de 1998, el Consejo de la Unión Europea decidió por unanimidad los 11 Estados miembros que cumplían las condiciones necesarias para participar en la tercera fase de la UEM y que querían adoptar la moneda única el 1 de enero de 1999. Dichos participantes iniciales fueron Bélgica, Alemania, España, Francia, Irlanda, Italia, Luxemburgo, Países Bajos, Austria, Portugal y Finlandia.

- El 25 de mayo de 1998, los Gobiernos de los 11 Estados miembros participantes nombraron al presidente, al vicepresidente y a los otros cuatro miembros del Comité Ejecutivo del BCE. La toma de posesión de estos cargos tuvo lugar el 1 de junio de 1998 y señaló la constitución del BCE. El BCE y los bancos centrales nacionales de los Estados miembros participantes constituyen el Eurosistema, que establece y formula la política monetaria única en la tercera fase de la UEM.

- Los ministros de Economía de los Estados miembros que habían adoptado la moneda única acordaron, junto con los gobernadores de los bancos centrales nacionales de dichos países, la Comisión Europea y el IME, que los tipos de cambio centrales bilaterales del SME de las monedas de los Estados miembros participantes serían utilizados para determinar los tipos de conversión irrevocable del euro.

\section{Tercera fase (1999-2002)}

La tercera fase comenzó el 1 de enero de 1999 con la fijación irrevocable de los tipos de cambio entre las monedas de los 11 Estados miembros que participaron inicialmente en la UEM, el inicio de la ejecución de la política monetaria única bajo la responsabilidad del BCE, y la adopción formal del euro como moneda única. La transición hacia el euro finalizó el 1 de enero de 2002, con la puesta en circulación de los billetes y monedas en euros. El número de Estados miembros participantes aumentó a 12, con la incorporación de Grecia, el 1 de enero de 20015.

En esta tercera fase entraron en vigor tanto el MTC II como el PEC.

${ }^{5}$ El resto de incorporaciones a la eurozona (Eslovenia, en 2007; Chipre y Malta en 2008; Eslovaquia, en 2009; Estonia en 2011; y Letonia en 2014) tienen lugar ya después de la ampliación de 2005. 
Durante esta etapa de construcción de la UEM se van consolidar unos principios fundamentales que modularán los avances futuros en la integración:

- El principio de subsidiariedad (introducido por el Tratado de Maastricht), por el cual en aquellas materias en las que las instituciones comunitarias no tienen la competencia exclusiva sólo podrá actuar si se demuestra que lo pueden hacer mejor que los Estados miembros, por la escala o los efectos de la acción propuesta. Y, además, este principio debía interpretarse conjuntamente con el de proporcionalidad, según el cual tanto el contenido como la forma de la acción comunitaria no deben exceder de lo que sea estrictamente necesario para conseguir los objetivos de los tratados (Department for Business, Innovation and Skills of the British Government, 2013, p. 5).

- El principio implícito de optatividad, según el cual es posible una Europa a la carta: Los Estados miembros podrán decidir la intensidad de su vínculo de integración y participar o no en los sucesivos avances hacia una política económica común.

Por otra parte, ya desde el proceso de su gestación, numerosos economistas $^{6}$ alertaron sobre las deficiencias estructurales en el diseño de la zona del euro, configurada por países con tejidos productivos y niveles de competitividad muy diversos y que no constituían una zona o área monetaria óptima ${ }^{7}$. Según la teoría de las Áreas Monetarias Óptimas (AMO), desarrollada por Robert Mundell (1961) $)^{8}$, cuando se dan las condiciones de una AMO se minimizan los costes derivados de su conformación, al tiempo que se alcanza un mayor aprovechamiento de sus beneficios asociados (Consejo Económico y Social español, 2012, p. 32).

6 «European Monetary Union remains fragile because of a flaw in its governance» (De Grauwe, 2006, p. 728).

${ }^{7}$ El diseño óptimo habría exigido uno de estos tres recursos: una gran movilidad del factor trabajo; una mayor flexibilidad de precios y salarios (posibilidad de devaluación interna); o, en ausencia de ambos, un grado de integración fiscal que permitiera suplir, al menos parcialmente, al tipo de cambio como mecanismo de ajuste ante un shock externo que afectara asimétricamente a distintos miembros de la Unión. Ninguna de estas tres condiciones, sin embargo, se da en la UEM: los dispositivos destinados a mitigar dichas carencias en lo relativo a prevenir desequilibrios dentro del espacio europeo, establecidos básicamente en el Pacto para la Estabilidad y el Crecimiento, no funcionaron (Noguer, 2011, p. 24). Adicionalmente, algunos autores (Toribio, 2011) afirman que España (quizá también Portugal, Grecia y otros países) entró en el esquema de moneda única a una paridad artificialmente baja, lo que condicionó la evolución posterior de estas economías.

8 «If the world can be divided into regions within each of which there is factor mobility and between which there is factor immobility, then each of these regions should have a separate currency which fluctuates relative to all other currencies» (Mundell, 1961, p. 664). 
Por tanto, para prevenir la aparición en la eurozona de shocks asimé$\operatorname{tricos}^{9}$ se requería, si no un mayor poder político común (un gobierno europeo con suficiente poder de imposición y gasto, es decir, dotado de un presupuesto compensatorio, como el Gobierno federal de los EE.UU., que pudo intervenir, por ejemplo, en la crisis de la burbuja inmobiliaria de $\mathrm{Ne}$ vada), sí al menos una coordinación reforzada en el resto de los ámbitos de la política económica. La racionalidad de este requerimiento era clara: ante crisis asimétricas, los países de la eurozona carecen ahora de herramientas convencionales de la política económica nacional para afrontarlas (como la manipulación del tipo de cambio o del tipo de interés, o la intervención del Banco central como instancia de último recurso ${ }^{10}$ ), y tampoco pueden esperar unas sustanciosas transferencias desde el Presupuesto común.

Efectivamente, como señala Bergsten (2012, p. 110), Europa fue construyendo una unión monetaria amplia, aunque incompleta, afianzada por el euro y el BCE, pero que no incluía prácticamente ninguna unión económica - ni fiscal ni bancaria, por ejemplo-, ninguna institución de gobernanza económica compartida, y ninguna coordinación eficaz de las políticas económicas estructurales.

Así, y dadas las restricciones derivadas del principio de subsidiariedad, se diseñaron distintos mecanismos e instrumentos destinados a mitigar las carencias institucionales de la eurozona, con el objeto de coordinar y supervisar multilateralmente las políticas macroeconómicas, fiscales, y de regulación y supervisión financiera, que permanecían en manos de los Gobiernos nacionales (Caballero, García y Gordo, 2011). Pero estos mecanismos se han manifestado fallidos y no han permitido ni diseñar una estrategia de salida de la crisis clara, ni instrumentar medidas suficientes para paliar los problemas sobrevenidos. Evidentemente, tampoco han servido para plasmar un esfuerzo de solidaridad entre los Estados miembros, algo que podría ir más allá de las reglas básicas de juego en la UE.

No cabe duda de que, en un ámbito puramente doctrinal, se requería a la gobernanza económica de la UE la posibilidad de disponer de mecanismos institucionales que permitieran dar respuesta en cada momento y con la suficiente flexibilidad, a las necesidades del mercado interior y la moneda única (Conde, 2004). Sin embargo, se fue construyendo un esquema de gobernanza

${ }^{9}$ La actual crisis europea respondería inicialmente a un perfil similar al del «shock asimétrico», al tratarse de un «shock simétrico con implicaciones asimétricas» (DG de Asuntos Económicos y Financieros de la Comisión Europea, 2009. p. 27-30). Y en la actual crisis del euro, sigue habiendo autores que sitúan en la construcción de la UEM, a partir del Tratado de Maastricht, las «raíces históricas» de los problemas actuales (Busch, 2012).

${ }_{10} \mathrm{Al}$ fin y al cabo, a estos efectos, es como si los países de la eurozona se endeudaran en moneda extranjera (De Grauwe, 2011). 
multinivel (Marks, 1993), por la que, hoy en día, instancias supranacionales definen algunas políticas (el BCE, por ejemplo, formula las políticas monetaria y cambiaria de la eurozona), mientras que otras son diseñadas o implementadas a nivel nacional - en cumplimiento del principio de subsidiariedad-, pero bajo dosis variables de coordinación o restricciones comunes a los Estados, en un esquema que se ha mostrado muy poco eficaz.

Esta «construcción asimétrica» (Rodríguez Ortiz, 2011) de la gobernanza económica (por la que se europeíza la política monetaria y se mantienen las demás competencias en materia económica en manos de los Estados) no fue atemperada por la dotación de recursos significativos al presupuesto europeo, ni por la previsión de mecanismos de asistencia inmediata en caso de urgentes necesidades financieras por parte de algún Estado miembro.

En todo caso, la concepción y posterior puesta en marcha de la UEM, a partir de los años 90, exacerbó la necesidad de coordinación, y por eso fueron surgiendo nuevos acuerdos y recursos de gobernanza económica europea en distintas dimensiones:

Compromisos en materia de disciplina fiscal:

- El Pacto de Estabilidad y Crecimiento (PEC), cuya primera versión fue aprobada en el Consejo Europeo de Ámsterdam (junio de 1997), y que luego fue modificado en 2005 (Reglamentos de la Comisión Europea 1055/2005 y 1056/2005) - añadiendo un procedimiento de aviso preventivo y de asesoramiento rápido- y en 2011 con la adopción del Six-Pack ${ }^{11}$. El objeto del PEC es fijar unos criterios cuantitativos necesarios para asegurar el mantenimiento de unas finanzas públicas saneadas de los Estados miembros y prever unos procedimientos de supervisión de su cumplimiento. Por tanto tiene una vertiente preventiva (los programas de estabilidad y convergencia que cada año presentan los Estados miembros, con sus objetivos presupuestarios y las perspectivas económicas, a la Comisión y al Consejo) y una vertiente correctora, que se basa en el procedimiento de déficit excesivo (al Estado miembro que no cumple el PEC se le pueden señalar recomendaciones e incluso imponer sanciones importantes).

Coordinación de las políticas económicas:

- Por un lado, la vertiente preventiva del PEC establece un mecanismo de supervisión multilateral (multilateral surveillance pro-

11 Paquete legislativo formado por 6 piezas de legislación (5 reglamentos y 1 directiva), 4 de las cuales incorporaban reformas de cierto alcance en el PEC. 
cedure) de los Programas de estabilidad (países de la zona euro) o de convergencia (el resto de los países), y desde 2005 de los Programas nacionales de reformas ${ }^{12}$. Esta supervisión es contemplada por el artículo 121 del Tratado de Funcionamiento de la UE (TFUE) y en el propio PEC, y fue posteriormente reformada (Reglamentos de la UE 1175/2011 y 1176/2011, aprobados dentro del Six-pack, que introducen un nuevo Procedimiento de Desequilibrios macroeconómicos); este mecanismo debe prevenir, en una fase inicial, la aparición de déficits excesivos de las administraciones públicas y promover la coordinación de las políticas económicas. El Consejo examina los programas nacionales al principio de cada año y emite un dictamen sobre cada uno de ellos, basándose en las evaluaciones de la Comisión y del Comité Económico y Financiero (CEF).

- Por otro, las Orientaciones Generales de Política Económica (OGPE), ya previstas en el art. 103-2 del Tratado de Maastricht, establecen, desde el Consejo Europeo de Lisboa (2000), unas líneas directrices para las políticas económicas de los Estados miembros. El Consejo Europeo las adopta en forma de recomendaciones no vinculantes jurídicamente. No obstante, se someten a un mecanismo de seguimiento multilateral que tiene por objeto garantizar su cumplimiento por parte de los Estados miembros.

Coordinación de las políticas sociales y de empleo:

- El Método Abierto de Coordinación (MAC), creado también a raíz del Consejo de Lisboa (2000), es un instrumento de coordinación de políticas entre los Estados miembros en áreas en las cuales la UE no puede legislar ya que no son de su competencia, como las políticas de empleo, las políticas de protección social o de inclusión social. Este método se basa en el intercambio de buenas prácticas y en la realización de evaluaciones y recomendaciones. Ha sido calificado por la propia Comisión (2008) como un «instrumento innovador de la gobernanza europea».

- En este marco se lleva a cabo la Estrategia Europea de Empleo, que se desarrolla a partir del Consejo Europeo extraordinario sobre el empleo celebrado en Luxemburgo (20 y 21 de noviembre

12 Que fueron el resultado de la revisión de la Estrategia de Lisboa en la Consejo Europeo de primavera de 2005. cada Estado miembro presenta su programa nacional de reformas antes de abril de cada año, con un horizonte de un mínimo de tres años y con actualizaciones anuales. En la actualidad, es la principal herramienta de la Estrategia Europa 2020. 
de 1997) y se formaliza en la Estrategia de Lisboa (2000). El elemento central de esta Estrategia es la aprobación de unas directrices de empleo, que recogen las prioridades y objetivos comunes para las políticas nacionales de empleo, a los que deben ajustarse los Programas Nacionales de Reforma (informes presentados por los gobiernos de los países miembros, que describen sus políticas de empleo) que analiza la Comisión y pueden dar lugar a recomendaciones (Cerezo, 2011) ${ }^{13}$.

Finalmente, se comienzan a adoptar decisiones comunes en el seno del Eurogrupo, que son reuniones de los ministros de economía de los países de la eurozona, que comenzaron como encuentros informales el 4 de junio de 1998, y cuyo papel es asegurar una más estrecha coordinación de las políticas económicas y facilitar la estabilidad financiera. En la actualidad el Eurogrupo se reúne al menos una vez al mes y en vísperas de las sesiones del ECOFIN.

Para algunos autores, en este esquema de arquitectura de gobernanza económica subyacía una visión liberal y ortodoxa de hacer política económica, orientada al objetivo prioritario de control de la inflación por el BCE, a la imperiosidad de las políticas fiscales severas y a la necesidad de reformas estructurales (Watt, 2011). Considerando la paulatina configuración de esa gobernanza multinivel europea, y específicamente el diseño de la zona del euro, advertimos que, tanto en los aspectos ideológicos como en los organizativos de esta arquitectura de gobernanza, se constatan unas deficiencias estructurales que limitaron su capacidad, primero para prevenir $\mathrm{y}$, segundo, para atajar la crisis actual.

\section{Los cambios en la gobernanza económica durante la gestión de la crisis}

La gestión de una crisis que ha estado a punto de desbordar a las instituciones comunitarias en varios momentos (crisis griega, contagio al resto de economías periféricas, crisis de deuda soberana y riesgo de ruptura del euro, crisis de Chipre, rescates urgentes, credit crunch generalizado, siste-

13 Por Decisión del Consejo, de 22 de abril de 2013, relativa a las orientaciones para las políticas de empleo de los Estados miembros, las orientaciones para las políticas de empleo de los Estados miembros expuestas en el anexo de la Decisión 2010/707/UE se mantienen para 2013 y los Estados miembros las tendrán en cuenta en sus políticas de empleo (Consejo de la UE, 2013a). 
mas financieros con problemas de solvencia, paro creciente, desafección por Europa) ha provocado la adopción de numerosas medidas de gobernanza económica europea, normalmente aisladas y que no han configurado una estrategia coherente, sólida y equilibrada de salida. Varios autores ${ }^{14}$ incluso imputan a esa insuficiente respuesta europea la responsabilidad principal de la recaída de la crisis a partir de 2010, cuando parecía superada la primera recesión.

En clave de economía política o de racionalidad inherente a las medidas planteadas, la estrategia oficial de salida de la crisis - apuntalada en 2011 - se ha basado en la exigencia de severos planes de austeridad y reformas estructurales a los países periféricos, además de en la preservación de una prudente política monetaria ajustada a la literalidad de los tratados, que luego - por fuerza de las apremiantes circunstancias - ha ido adquiriendo mayores grados de libertad.

Repasamos en este apartado las principales innovaciones introducidas en el esquema de intervención económica de la UE a raíz de la crisis, agrupándolas en 4 áreas:

\section{A) Cambios en materia de estabilidad financiera}

1. Se llevan a cabo los Planes de rescate de Grecia (2010), Irlanda (2010), Portugal (2011), y Chipre (2013), precedidos en cada caso del correspondiente Memorándum de Entendimiento (Memorandum of Understanding o MoU). También se aprueba el rescate bancario a España (2012) con un menor nivel de condicionalidad. Previamente, en mayo de 2010, el BCE ya comenzó a adquirir bonos de Grecia, Irlanda y Portugal en el mercado secundario, a raíz de la creación del «Programa de Mercado de Valores» o «Securities Markets Program» (SMP) (Banco Central Europeo, 2010), por el que se comprometía a adquirir en firme valores, públicos o privados, si ello resultaba necesario para evitar el colapso de ciertos segmentos de los mercados de deuda. El argumento era que ello imposibilitaría la transmisión de las señales de política monetaria.

2. Se realizan dos subastas de liquidez (Quantitative Easing) a tres años, con ampliación del colateral aceptado para los préstamos bancarios y rebaja del requerimiento de reservas desde el $2 \%$ al $1 \%$. En diciembre de 2011 se realizó la primera de las dos subastas de liquidez a tres años, por la que 523 bancos europeos obtuvieron 489.190 millones de euros al $1 \%$. La segunda subasta a 36 meses se llevó a cabo el 28 de febrero de 2012, y el

14 Vid., por ejemplo, DÍEZ, J.C., Hay vida después de la crisis, Plaza y Janes editores, Barcelona, 2013; BILBAO-UBILLOS, J. (ed.), The Economic Crisis and Governance in the European Union: a critical assessment, Routledge, Londres, 2014. 
volumen adjudicado ascendió a 529.531 millones de euros, a repartir entre 800 bancos de la eurozona (se estima que un $20 \%$ correspondió a entidades españolas).

3. Para garantizar la estabilidad financiera, se ha ido diseñando un Mecanismo de Estabilización Europeo. Primeramente, se aprobó el Reglamento (UE) n. ${ }^{\circ}$ 407/2010, de 19 de mayo de 2010, estableciendo el Fondo de Estabilidad Financiera (ESF), que se basa en el artículo 122-2 del TFUE y cuenta con 60.000 millones de euros, (financiado íntegramente por la Comisión contra su presupuesto ordinario); este Fondo se unía al Fondo Europeo de Estabilidad Financiera (EFSF) ${ }^{15}$ creado el 9 de mayo de 2010 y dotado con un máximo de 440.000 millones de euros.

Después, el 1 de julio de 2012, se puso en marcha el Mecanismo Europeo de Estabilidad (MEDE) - llamado a reemplazar a los anteriores que es una institución financiera internacional sujeta al derecho internacional, con sede en Luxemburgo, y cuyos accionistas son los 17 Estados miembros de la Unión Europea cuya moneda era el euro. El MEDE cuenta con 80.000 millones de euros de capital desembolsado y dispone de un capital suscrito de 700.000 millones de euros. Este capital le confiere una capacidad de préstamo efectiva de 500.000 millones de euros. El MEDE es un mecanismo permanente de resolución de crisis económicas para países de la zona euro. Su finalidad es movilizar fondos y proporcionar apoyo a la estabilidad financiera, bajo una estricta condicionalidad, a los miembros del MEDE que experimenten o corran el riesgo de experimentar graves problemas de financiación, cuando ello sea indispensable para salvaguardar la estabilidad financiera de la zona euro en su conjunto o de sus Estados miembros. El MEDE se estrenó con el rescate a Chipre (marzo de 2013).

4. Por acuerdo del Consejo de Gobierno del BCE, de 6 de septiembre de 2012, se diseña un cortafuegos financiero basado en operaciones directas del Eurosistema en los mercados secundarios de bonos soberanos (anunciado previamente el 2 de agosto). Se trata de operaciones monetarias directas - para el BCE, Outright Monetary Transactions (OMT); para el Banco de España, Transacciones monetarias al contado (TMC) - por las que el $\mathrm{BCE}$ se compromete a comprar bonos soberanos con vencimiento de uno a

15 La EFSF es una sociedad anónima de carácter instrumental y temporal constituida en Luxemburgo el 7 junio de 2010, según el acuerdo del ECOFIN de 9 de mayo del mismo año por los entonces 16 Estados miembros de la zona euro, a los que posteriormente se uniría Estonia, tras incorporarse el 1 de enero de 2011 a la eurozona. Actualmente, los Estados beneficiarios de la asistencia financiera de la EFSF son:

- Con Programa de ajuste: Grecia, Irlanda y Portugal.

- Sin programa de ajuste: España. 
tres años con carácter ilimitado ${ }^{16}$, pero sometida la intervención a condicionalidad: será necesario que el país que necesite asistencia lo solicite, que se firme un MoU con unas condiciones a cumplir, y que los fondos de rescate europeos actúen comprando en el mercado secundario. Las OMT mejoran la capacidad de intervención del SMP creado en mayo de 2010 y que cesa al ser sustituido por estas operaciones. Sin embargo, y fiel a la actitud prudente que ha mostrado siempre el BCE, se contempla que la liquidez derivada de la adquisición de los títulos sea plenamente esterilizada para evitar riesgos de inflación.

\section{B) Cambios en materia de supervisión financiera}

1. Se aprueba en septiembre de 2010 el Nuevo Marco Europeo de Supervisión Financiera, y se decide avanzar hacia el Mecanismo único de Supervisión bancaria (MUS). En 2011 comienzan a operar tres nuevas autoridades europeas de supervisión (Autoridad Bancaria Europea, Autoridad Europea de Valores y Mercados, y Autoridad Europea de Seguros y Pensiones de Jubilación) para evitar la acumulación de riesgos que pongan en peligro la estabilidad del sistema financiero general, y surge otra (Junta Europea de Riesgo Sistémico) que sustituye a los anteriores comités de supervisión.

2. En junio de 2012, los líderes europeos deciden que debería crearse un MUS como parte de un más amplio esfuerzo para lograr la futura Unión bancaria, profundizando así en la UEM. Bajo el MUS - sobre el que la Comisión planteó ya una propuesta concreta en septiembre de 2012- el BCE y los supervisores nacionales se coordinarán estrechamente para supervisar las instituciones bancarias de la eurozona, además de las de los países que, sin pertenecer a la misma, participen en el mecanismo. Una vez que sea plenamente operativo los fondos de estabilidad podrán emplearse en la recapitalización directa de la banca.

3. La Comisión Europea lanza el 10 de julio de 2013 una propuesta de Mecanismo único de Resolución (MUR) en el marco de la senda hacia dicha Unión bancaria, completando el MUS que entrará en funcionamiento a finales de 2014 (aunque sólo supervisará las 130 entidades de mayor tamaño de la eurozona). El MUR garantizará que, si un banco sujeto al MUS se enfrentase a graves dificultades, pudiera procederse a su resolución de manera ágil y con un coste mínimo para los contribuyentes y la economía real. El Consejo Europeo de 19-20 de diciembre de 2013 aprobó una ver-

16 Según parece, las compras se realizarían de manera masiva durante uno o dos meses seguidos, y luego se valorarían las necesidades y los cumplimientos del acuerdo por parte del país rescatado. 
sión muy aligerada de compromiso sobre el MUR y el Fondo de liquidación para satisfacer las exigencias alemanas. Estos son los elementos centrales del acuerdo:

- El MUR empezará a funcionar en 2016, y para ello los países participantes (en un principio, los miembros de la zona del euro más aquellos que se unan voluntariamente) se comprometen a firmar un tratado internacional sobre el que se asentará el fondo de resolución;

- La decisión de liquidación será tomada por representantes de los Estados miembros, teniendo la Comisión un papel secundario aunque sea preceptiva su conformidad;

- El Fondo de liquidación se nutrirá de aportaciones anuales de 5.500 millones de euros procedentes de la banca durante 10 años, y estará dividido en compartimentos estancos nacionales (el español, por ejemplo, alcanzará al final los 8.500 millones de euros), hasta que en 2026 se haya alcanzado el total de 55.000 millones de euros. Es decir, durante los próximos 10 años cada país sufragará con sus recursos nacionales las necesidades financieras que surjan por entidades residentes abocadas a quiebras o reestructuraciones, usando su fondo nacional o recurriendo eventualmente al MEDE.

Dado el poder de codecisión con que cuenta el Parlamento europeo (PE) en este asunto, este acuerdo deberá ser refrendado por la Cámara a principios de 2014. El propio presidente del PE se ha quejado tanto del papel secundario al que se relega a la Comisión en el MUR como de la resistencia a asumir una mayor mutualización del riesgo de quiebra bancaria a través de un fondo común. Numerosos autores desconfían de que, en estos términos, pueda hablarse de una genuina Unión bancaria, habiéndose rebajado notablemente las expectativas surgidas a raíz de la cumbre de junio de 2012.

\section{C) Cambios en materia de coordinación de políticas económicas}

1. Con los cambios introducidos, se han configurado al respecto espacios diferenciados de Gobernanza económica en la UE, y podríamos hablar de una coordinación de políticas económicas a distintas velocidades:

Ámbito 1: Países de la zona euro (18 de los 28 Estados miembros): En estos países, además de la normativa básica común, se han asumido compromisos adicionales:

- Por un lado, el BCE define la política monetaria y cambiaria para esta eurozona, y está llamado a desempeñar un papel esencial en los mecanismos de supervisión financiera. El MUS y el MUR, en principio, regirán para este grupo de países. 
- Por otro, se refuerza la coordinación de las políticas presupuestarias: el Consejo de la Unión europea celebrado el 13 de mayo de $2013^{17}$ adoptó formalmente un nuevo paquete legislativo, denominado en la jerga europea Two-Pack ${ }^{18}$, que otorga nuevos poderes a la Comisión Europea en la supervisión económica de la zona euro. El Two-Pack presenta como novedad más llamativa la obligatoriedad de remitir a la Comisión Europea los anteproyectos de Presupuestos nacionales a mediados de octubre de cada año. La Comisión examinará y emitirá un dictamen sobre cada uno de los proyectos de presupuestos; en caso de grave incumplimiento de un plan con las obligaciones contraídas en virtud del PEC, la Comisión podría solicitar que se presente un nuevo proyecto de presupuesto. El Reglamento n. ${ }^{\circ}$ 473/2013 impone también a los Estados miembros de la zona del euro la obligación de comunicar, ex ante y oportunamente, sus planes nacionales de emisión de deuda; asimismo los proyectos de planes presupuestarios también deberán informar sobre la incidencia distributiva prevista de las principales medidas de gastos e ingresos.

- A su vez las reuniones del Eurogrupo, que a raíz de la crisis actual han adquirido un carácter crecientemente decisorio. En estas reuniones los ministros de los Estados miembros que han adoptado el euro como moneda examinan cuestiones vinculadas a las responsabilidades específicas que comparten en lo relativo a la moneda única, y han acordado, por ejemplo, la constitución y dotación de fondos de rescate como el MEDE. El TECG reconoce formalmente el papel de las Cumbres del euro (art. 12).

- Finalmente, el Consejo Europeo de diciembre de 2013 fija para octubre de 2014 la puesta en marcha del sistema de contratos de reformas que deberán suscribir los países de la eurozona a cambio de un apoyo financiero muy limitado de la UE (pensado para países con dificultades momentáneas para acceder a la financiación en los mercados). El nuevo sistema de los contratos de reformas (propuesto por Alemania) pretende reforzar la coordinación de políticas económicas

17 El Parlamento europeo lo había aprobado el 12 de marzo de 2013.

18 Son dos reglamentos: Reglamento (UE) No 473/2013 del Parlamento europeo y del Consejo, de 21 de mayo de 2013, sobre disposiciones comunes para el seguimiento y la evaluación de los proyectos de planes presupuestarios y para la corrección del déficit excesivo de los Estados miembros de la zona del euro. Diario Oficial de la Unión Europea L 140/11 de 27-5-2013; Reglamento (UE) No 472/2013 del Parlamento europeo y del Consejo, de 21 de mayo de 2013 sobre el reforzamiento de la supervisión económica y presupuestaria de los Estados miembros de la zona del euro cuya estabilidad financiera experimenta o corre el riesgo de experimentar graves dificultades. Diario Oficial de la Unión Europea L 140/11 de 27-5-2013. 
y la vigilancia sobre los Estados miembros con el fin de prevenir futuras crisis. Estos contratos serán redactados por los propios países, con un calendario estricto para las reformas, luego se negociarán con la Comisión y los aprobará definitivamente el ECOFIN. Se centrarán en las áreas donde podría haber obstáculos clave para el crecimiento sostenible, la competitividad y la creación de empleo y que representan un riesgo potencial para la eurozona en su conjunto.

Ámbito 2: Países firmantes el Pacto por el euro-Plus (23 de los 28): Estos países, entre los que se encuentran todos los del colectivo anterior, han aceptado compartir esos objetivos:

- Impulso de la competitividad a evolución de los salarios y la productividad y las necesidades de ajuste de la competitividad.

- Impulso del empleo reformas del mercado laboral para fomentar la «flexiseguridad», educación permanente; reformas fiscales, tales como la reducción de la presión impositiva sobre las rentas del trabajo

- Incremento de la sostenibilidad de las finanzas públicas, y en especial de las pensiones, la atención sanitaria y las prestaciones sociales en general.

- Refuerzo de la estabilidad financiera reforma completa del marco de la UE para la supervisión y la reglamentación del sector financiero.

- Se prestará atención a la coordinación de la política tributaria.

Los compromisos anuales concretos se reflejarán también en los programas nacionales de reforma y en los programas de estabilidad o convergencia que se presenten cada año, que serán evaluados por la Comisión, el Consejo y el Eurogrupo en el contexto del Semestre Europeo.

Ámbito 3: Países firmantes del Tratado de Estabilidad, Coordinación y Gobernanza en la UEM (TECG o Fiscal Compact), suscrito por 25 países (los anteriores y dos más), el 2 de marzo de 2012, y que entró en vigor el 1 de enero de 2013. El TECG contiene un conjunto de reglas de disciplina fiscal, llamadas «reglas de oro»:

- Un compromiso con el equilibrio presupuestario, entendido como el logro de un saldo estructural anual superavitario o de un déficit inferior al 0,5\% del PIB, salvo circunstancias excepcionales y, en todo caso, con carácter temporal; cuando la proporción entre la deuda pública y el PIB a precios de mercado esté muy por debajo del $60 \%$ y los riesgos para la sostenibilidad a largo plazo de las finanzas públicas sean bajos, se admitirá un déficit estructural máximo del $1 \%$. Este compromiso debería ser constitucionalizado por los países firmantes. 
- Cada Estado deberá incorporar a su legislación un mecanismo automático de reequilibrio presupuestario en el caso de que se superen los máximos déficits citados anteriormente.

- La obligación de los países con una deuda pública superior al $60 \%$ del PIB a bajarla de ese límite en 20 años, a una tasa igual a la veinteava parte del diferencial en cada anualidad.

- Esta nueva versión del PEC, por un lado, permite un mínimo juego de los estabilizadores automáticos (se adopta como indicador el saldo presupuestario anual ajustado en función del ciclo), y, por otro, - relevante en clave de gobernanza - introduce un criterio de «mayoría inversa»: a partir de ahora hará falta rechazar por mayoría la adopción de mecanismos correctores o sancionadores propuestos por la Comisión Europea, mientras que en el PEC era necesaria alcanzar la mayoría para aprobar esas propuestas (Andrés y Domenech, 2012).

Ámbito 4: Conjunto de la UE: los 28 países miembros (con la incorporación de Croacia el 1 de julio de 2013) que están vinculados por:

- El PEC y el Six-pack cuyas clausulas regulan la supervisión de las situaciones presupuestarias y coordinación de las políticas económicas; el procedimiento de déficit excesivo; y la detección y combate eficaz de los desequilibrios macroeconómicos aparecidos en la UE. En efecto, el Six-pack introduce un nuevo Procedimiento de Desequilibrios macroeconómicos, que debe prevenir, en una fase inicial, la aparición de déficits excesivos de las administraciones públicas y promover la coordinación de las políticas económicas. El Consejo examina los Programas nacionales de estabilidad o convergencia, y los Programas nacionales de reformas (PNR) ${ }^{19}$ al principio de cada año y emite un dictamen sobre cada uno de ellos, basándose en las evaluaciones de la Comisión y del Comité Económico y Financiero (CEF). El año 2012 se ha iniciado ya la aplicación de este nuevo procedimiento de vigilancia adicional, para la prevención y corrección de desequilibrios macroeconómicos (Comisión Europea, 2012).

- La nueva Estrategia para el crecimiento y el empleo (Europa 2020) - aprobada el 26 de marzo de 2010 por el Consejo Europeo a propuesta de la Comisión-, que se basa en una mayor coordinación de las políticas económicas y se centra en los ámbitos fundamentales en que se requiere actuar para fomentar el potencial de crecimiento

19 España presentó su último PNR el 30 de abril de 2013, junto con el Programa de Estabilidad para el período 2012-2016; ambos fueron dictaminados por el Consejo el 29 de mayo de 2013 (Consejo de la UE, 2013b). 
sostenible y competitividad de la economía europea. Con este fin, se aprobó un nuevo método de trabajo para mejorar la coordinación de las políticas económicas, el Semestre Europeo, que pretende sincronizar los calendarios de la evaluación y los informes de la política económica y presupuestaria a nivel de la UE, y cambiar la coordinación de las políticas económicas nacionales de ex post a ex ante. Para ello se establecen unas etapas:

- En enero, la Comisión presenta su Estudio Prospectivo Anual sobre el Crecimiento (EPAC), que establece las prioridades de la UE para impulsar el crecimiento y la creación de empleo durante el año que empieza.

- En marzo, los Jefes de Estado o de Gobierno de la UE presentan las orientaciones de la UE para las políticas nacionales, basadas en el EPAC.

- En abril, los Estados miembros presentan sus planes para conseguir unas finanzas públicas saneadas (Programas de Estabilidad o Convergencia), así como reformas y medidas para alcanzar un crecimiento inteligente, sostenible e integrador (Programas $\mathrm{Na}$ cionales de Reforma).

- En junio, la Comisión evalúa esos programas y presenta recomendaciones específicas por país, si procede. El Consejo debate estas recomendaciones y el Consejo Europeo las aprueba seguidamente.

- Por último, a finales de junio o principios de julio, el Consejo adopta oficialmente las recomendaciones específicas para cada país.

2. Las nuevas Directrices generales para las políticas económicas de los Estados miembros y de la Unión (para el período 2010-2014), aprobadas por el Consejo mediante una Recomendación de 13 de julio de 2010, basándose en las propuestas de la Comisión; el 21 de octubre de 2010 se adoptó una decisión relativa a las Orientaciones para las políticas de empleo de los Estados miembros. Ambas directrices forman las orientaciones integradas que deben ser tenidas en cuenta en la formulación nacional de las políticas económicas y de empleo.

\section{D) Cambios en las políticas de crecimiento}

1. En 2010, la UE adoptó una serie de objetivos comunes para promover el crecimiento en la citada Estrategia Europa 2020, que aspira a proporcionar un marco para el crecimiento inteligente, sostenible e inclusivo.

2. Adicionalmente, en el Consejo Europeo de junio de 2012, los Jefes de gobierno de la UE aprobaron un Pacto para el crecimiento y el empleo 
(PCE), orientado a la recuperación del crecimiento, la inversión y el empleo y a hacer más competitiva la economía europea. Se preveía llegar a movilizar hasta 120.000 millones de euros para inversiones inmediatas en el conjunto de la UE.

¿Por qué interpretábamos que estas medidas no formaban parte de un esquema coherente y equilibrado? Porque, desde un punto de vista doctrinal, la cesión de soberanía en materia presupuestaria (incluso permitiendo que la Comisión estudie los proyectos de presupuestos nacionales en la eurozona y formule recomendaciones) serviría de contrapunto a un refuerzo de la solidaridad con avances hacia una Unión fiscal (a través de un más dotado y potencialmente compensatorio Presupuesto comunitario; imprimiendo un carácter más expansivo a sus políticas fiscales los países centrales que cuentan con margen para ello), o al logro de una Unión bancaria con un fondo europeo de garantía de depósitos. Es decir, se exigiría más responsabilidad a los países (evitando el moral hazard) para ofrecer a cambio más solidaridad o avances en la federalización de la UE (Unión fiscal o Unión bancaria).

Pues bien, la cesión de soberanía (que ha supuesto también la inclusión de la regla de oro presupuestaria en la legislación fundamental de los Estados miembros ${ }^{20}$, y el envío de misiones de la troika a los países rescatados con propuestas de medidas y reformas) no ha sido premiada, hoy en día, con decisivos avances hacia la Unión fiscal (EUROPEG, 2012) o la Unión bancaria:

- el PCE no se ha puesto en marcha aún;

- se aprueba - por primera vez - una reducción del montante del presupuesto plurianual comunitario ${ }^{21}$;

- no se avanza significativamente en la armonización fiscal;

- los países centrales de la UE siguen apostando por un crecimiento virtuoso vía exportaciones, sin activar sus políticas fiscales;

- y se ha optado, inicialmente, en el Consejo Europeo de diciembre de 2013 por una regulación del MUR y del Fondo de liquidación que debilita el alcance de la Unión bancaria ${ }^{22}$.

${ }^{20}$ Recuérdese la modificación de la Constitución española con este objetivo.

${ }^{21}$ El Consejo de la Unión europea aprobó formalmente, el 2 de diciembre de 2013, el Presupuesto plurianual comunitario de 2014 a 2020. Este acuerdo presupuestario para los próximos siete años fija en 959.988 millones de euros los compromisos - el importe máximo de pagos futuros a los que se puede comprometer la UE - y en 908.400 millones los pagos, lo que supone un $3,5 \%$ y un 3,7 \% menos en comparación con el marco plurianual de 2007 a 2013. Por vez primera su cuantía porcentual baja del $1 \%$ del PIB comunitario, situándose en el $0,95 \%$ en créditos de pago.

22 El Parlamento Europeo aprobó el 12 de septiembre de 2013, el Reglamento del Consejo por el que se establece el Mecanismo único de supervisión (MUS) - el Reglamento MUS - que puede ser publicado y entrar en vigor en un futuro próximo. La francesa Danièle 
Al margen de estas consideraciones, hoy casi nadie duda de que las políticas de austeridad recetadas en la UE en respuesta a la crisis han condicionado la pobre evolución económica de la UE en los últimos años: a los reconocimientos del FMI (2013) y de varios autores (Vid. Semmler y Semmler, 2011; De Grauwe y Ji, 2013) de que, en el diseño de las políticas de austeridad en la UE, se subestimaron los multiplicadores fiscales, se ha añadido recientemente el informe de un experto de la Comisión Europea (In't Veld, 2013), que cuantifica las perdidas de crecimiento económico para el conjunto de países de la UE (no sólo de los que se vieron obligados a emprender severos planes de ajuste) por los significativos spillovers negativos desencadenados (spillovers de demanda, de competitividad y de flujos financieros internacionales).

\section{Balance y conclusiones}

La evolución de la arquitectura de gobernanza económica europea no ha sido lineal, ni ha obedecido la mayoría de las veces a un esquema secuencial ordenado, sino que ha experimentado una serie de cambios estructurales que han permitido avanzar aparentemente hacia una política económica común, coincidiendo normalmente con etapas expansivas del ciclo económico, con la excepción de la crisis, cuya gestión reciente ha provocado notables novedades en el esquema de intervención económica en la UE.

La transición del mercado común inicial hacia una comunidad económica, y, posteriormente, hacia una Unión monetaria (aún parcial) no ha conducido a la convergencia económica, ni siquiera a la sincronización de las economías europeas; tampoco a la concertación de los planteamientos de política económica (el componente ideológico de la gobernanza). La dispar evolución de las economías de los Estados miembros, apoyada en diferentes modelos de crecimiento (basado en el aprovechamiento del potencial exportador en los países centrales; anclado en la expansión de la demanda interna - aprovechando los bajos tipos de interés - en los países periféricos), ha favorecido la aparición de fuertes desequilibrios comerciales en el interior de

Nouy fue propuesta por Mario Draghi como presidenta del MUS el 20 de noviembre de 2013, pendiente de ser nombrada formalmente en un próximo Consejo de la Unión europea. Sin embargo, el acuerdo de mínimos sobre el MUR alcanzado en diciembre de 2013 no satisface - aparentemente - las expectativas de la Comisión y el Parlamento Europeo. El BCE comunicó recientemente que el MUR encaja perfectamente en el artículo 114 del TFUE, en línea con la Comisión Europea y en contra de lo que ha argumentado Alemania, que interpreta que el mecanismo es una forma de que los países más solventes financien a los países con problemas a través de los bancos. 
la UE, que han añadido complejidad a la definición de una política económica común, dados los diferentes intereses y coyunturas nacionales.

Además, en la etapa de construcción de la UEM se consolidaron unos principios fundamentales como los de subsidiariedad y proporcionalidad, y el principio implícito de optatividad, según el cual es posible una Europa a la carta: Los Estados miembros podrán decidir la intensidad de su vínculo de integración en algunos aspectos (como las políticas monetaria y cambiaria, o la supervisión de las políticas económicas y, especialmente, de la disciplina fiscal) y participar o no en los sucesivos avances hacia una política económica común, con sus correspondientes cesiones de soberanía.

Esta complejidad creciente, unida al sesgo inicial de primar ante todo la construcción de un Mercado interior - en el que las empresas aprovechasen economías de escala y alcance, y donde se asignaran más eficientemente los bienes, factores productivos y capitales - ha provocado la actual construcción asimétrica de la gobernanza económica europea:

- se ha logrado la comunitarización plena de las políticas comercial y agrícola-pesquera, y se han sometido a fiscalización las políticas industriales nacionales;

- se han unificado las políticas monetaria y cambiaria para los países de la eurozona, mientras que se mantienen acuerdos con los Estados miembros cuyas monedas nacionales aún perviven a través del MTC II;

- los países de la eurozona cuentan con un Mecanismo europeo de estabilidad específico (MEDE), además del cortafuegos financiero inherente a las operaciones OMT del BCE;

- se está configurando un Mecanismo único de supervisión financiera para los países de la eurozona, al que podrán sumarse otros Estados miembros;

- la coordinación de la política fiscal se efectúa a 4 niveles distintos de exigencia, en función de los acuerdos suscritos por los Estados miembros;

- la regulación de los mercados e instituciones y de la política social (que incide en muchas dimensiones de la competitividad) sigue formulándose esencialmente a nivel nacional, en el marco del MAC, y sólo los países rescatados han sufrido injerencias comunitarias de entidad;

- el Presupuesto comunitario para financiar las políticas comunes equivaldrá a partir de ahora al 0,95\% del PIB de la UE, lo que da idea de su insuficiencia para operar con un carácter compensatorio.

Este complejo y asimétrico sistema de interrelaciones estaría reflejando que el denominador común del proyecto europeo - lo realmente compar- 
tido como aspiración de la integración por todos los países - ha sido la construcción plena del Mercado interior, para lo que se han adoptado todas las medidas consideradas necesarias, aunque supusieran compromisos adicionales en determinadas políticas económicas (monetaria-cambiaria o fiscal, por ejemplo). Por eso suele afirmarse, fundadamente, que se ha subordinado el proyecto de construcción europeo $-\mathrm{y}$ sobre todo su dimensión social - a las metas económicas compartidas.

En síntesis, y hablando en términos de economía política, lo que subyace a lo largo del proceso de construcción de la UE es una absoluta confianza en el mercado como mecanismo asignativo óptimo y favorecedor de la convergencia real, desde un enfoque sesgado hacia la oferta y cicatero en la administración de la solidaridad entre los Estados miembros, por el débil sentido de pertenencia a Europa, la desconfianza respecto a los demás países y la precaución sobre el «riesgo moral». Y con ocasión de la crisis - que ha supuesto un desafío imprevisto que ha convulsionado la arquitectura de gobernanza económica europea - se ha perdido una oportunidad única para avanzar hacia la federalización de la UE, porque las cesiones de soberanía de muchos países no se han correspondido con pasos significativos hacia la Unión fiscal o la Unión bancaria, por ejemplo, con la consiguiente mutualización de riesgos.

\section{Bibliografía}

ANDRÉS, J. y DOMENECH, R., «El futuro del euro tras la Gran recesión», en $E l$ futuro del euro. Libro marrón 2012, Círculo de empresarios, Madrid, 2012, pp. 15-66.

BERGSTEN, F., «Por qué el euro sobrevivirá» en Política Exterior, Vol. 26 (150), 2012, pp. 110-120.

BILBAO-UBILLOS, J. (ed.), The Economic Crisis and Governance in the European Union: a critical assessment, Routledge, Londres, 2014.

BUSCH, K., Is the Euro Failing? Structural Problems and Policy Failures Bringing Europe to the Brink, International Policy Analysis-Friedrich-Ebert-Stiftung, Berlín, 2012.

CABALLERO, J.C., GARCÍA, P. y GORDO, E., «La reforma de la gobernanza económica en la UEM», en Boletín económico del Banco de España de Enero de 2011, 2011, pp. 118-134.

CEREZO, C. J., Estrategia europea de empleo, Trabajo fin de grado en relaciones laborales y recursos humanos, Facultad de Ciencias del Trabajo de Palencia, Universidad de Valladolid, 2011.

COMISIÓN DE LAS COMUNIDADES EUROPEAS, Crecimiento, competitividad y empleo. Retos y pistas en el siglo XXI. Libro Blanco, Boletín de las Comunidades europeas Suplemento 6/1993, Oficina de Publicaciones oficiales de las Comunidades europeas, Luxemburgo, 1993. 
COMISIÓN EUROPEA, Comunicación de la Comisión al Parlamento europeo, al Consejo, al Comité económico y social europeo y al Comité de las regiones «Un compromiso renovado en favor de la Europa social: reforzar el Método abierto de coordinación en al ámbito de la protección social y la inclusión social», COM (2008) 418 final, (Bruselas, 2/7/2008).

COMISIÓN EUROPEA, Report from the Commission: Alert Mechanism Report. Report prepared in accordance with Articles 3 and 4 of the Regulation on the prevention and correction of macro-economic imbalances, COM (2012) 68 final, (Bruselas, 14/2/2012).

CONDE, F., «Constitución europea y gobernanza económica», en Quaderns de Po-

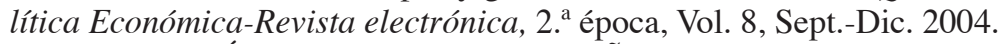

CONSEJO ECONÓMICO Y SOCIAL ESPAÑOL, La nueva gobernanza económica en la Unión europea y crecimiento, Informe 0312012, CES, Madrid, 2012.

CONSEJO DE LA UE, Decisión del Consejo, de 22 de abril de 2013, relativa a las orientaciones para las políticas de empleo de los Estados miembros (2013/208/ $U E)$, Diario Oficial de la UE, 30-4-2013, 2013a.

CONSEJO DE LA UE, Recomendación del Consejo, de 29 de mayo de 2013, relativa a la aplicación de las orientaciones generales de política económica por los Estados miembros cuya moneda es el euro, COM (2013) 379 final, 2013b.

DE GRAUWE, P., «The Governance of a Fragile Eurozone», en Centre for European Policy Studies Working Paper, no 346, CEPS, Bruselas, 2011.

DE GRAUWE, P., «What have we learnt about monetary union since the Maastricht Treaty?» en Journal of Common Market Studies, Vol. 44, 2006, pp. 711-30.

DE GRAUWE, P. y JI, Y., «Panic-driven austerity in the Eurozone and its implications», en VoxEu, February, 21th, 2013.

DÍEZ, J. C., Hay vida después de la crisis, Plaza y Janes Editores, Barcelona, 2013.

DG DE ASUNTOS ECONÓMICOS Y FINANCIEROS DE LA COMISIÓN EUROPEA, Economic Crisis in Europe: Causes, Consequences and Responses. European Economy 7/2009, Office for Official Publications of the European Communities, Luxemburgo, 2009.

DEPARTMENT FOR BUSINESS, INNOVATION AND SKILLS OF THE BRITISH GOVERNMENT, Review of the balance of competences between the United Kingdom and the European Union, Call for Evidence: Social and Employment Review, October 2013, British Government, Londres, 2013.

EUROPEG, «¿Avanza realmente la Unión Europea hacia una Unión Fiscal?», en Policy Brief, N. ${ }^{\circ}$ 3, EUROPEG, Barcelona, 2012.

FMI, Growth Forecast Errors and Fiscal Multipliers, FMI, Washington, 2013.

GHYMERS, C., La problemática de la coordinación de políticas económicas, CEPAL, Santiago de Chile, 2001.

IN'T VELD, J., «Fiscal consolidations and spillovers in the Euro area periphery and core», en European Economy-Economic Papers, n. ${ }^{\circ}$ 506, October 2013, Comisión Europea, Bruselas, 2013.

MARISCAL, N., «La gobernanza en las políticas del Tratado Constitucional de la Unión Europea», Comunicación presentada al VII Congreso Español de Ciencia Política y de la Administración: Democracia y Buen Gobierno, Madrid, 21-13 de septiembre de 2005. 
MARKS, G., «Structural Policy and Multilevel Governance in the EC», en CAFRUNY, A. W. y ROSENTHAL, G. (eds.), The State of the European Community (Vol. 2: The Maastricht Debates and Beyond), Boulder Publishers/Harlow, Boulder, 1993.

MUNDELL, R., «The theory of optimum currency areas» en American Economic Review, Vol. 51 (4), 1961, pp. 657-665.

NOGUER, M., «Eurozona: remedios, no parches», en Informe mensual, n. ${ }^{\circ}$ 352, Estudios y Análisis Económico de La Caixa, Barcelona, 2011, pp. 23-25.

PARLAMENTO EUROPEO Y CONSEJO DE LA UNIÓN EUROPEA, Reglamento (UE) No 1176/2011 del Parlamento europeo y del Consejo, de 16 de noviembre de 2011, relativo a la prevención y corrección de los desequilibrios macroeconómicos.

PARLAMENTO EUROPEO Y CONSEJO DE LA UNIÓN EUROPEA, Reglamento (UE) No 1175/2011 del Parlamento europeo y del Consejo, de 16 de noviembre de 2011, por el que se modifica el Reglamento (CE) no 1466/97 del Consejo, relativo al refuerzo de la supervisión de las situaciones presupuestarias y a la supervisión.

RODRÍGUEZ ORTIZ, F., «Crisis de la deuda pública: ajuste social regresivo y nueva gobernanza económica asimétrica», en Revista de Economía mundial, n. ${ }^{\circ} 29,2011$, pp. 179-207.

SEMMLER, W. y SEMMLER, A., The Macroeconomics of the Fiscal Consolidation in the European Union, Lecture delivered by Professor Semmler in celebration of Willi Semmler's appointment as the Henry H. Arnhold Professor of International Cooperation and Development in the Department of Economic at The New School for Social Research, New York, 19/03/2013.

TORIBIO, J. J., «Áreas monetarias óptimas y la experiencia europea: algunas reflexiones», en Información Comercial Española-Revista de Economía, n. ${ }^{\circ}$ 863, 2011, pp. 13-19.

VAN MIDDELAAR, L., El paso hacia Europa. Historia de un comienzo, GalaxiaGutemberg, Barcelona, 2013. (Traducción del original: De passage naar Europa. Geschiedenis van een begin, Historische Uitgeverij, Groningen, 2009).

WATT, A., «Economic governance in Europe in the wake of the crisis: reform proposals and their alternatives», en European Review of Labour and Research, Vol. 17 (2), 2011, pp. 255-261. 